УдК 621.314

\title{
Алексей Васильевич Гусенков
}

ФГБОУВО «Ивановский государственный энергетический университет имени В.И. Ленина», кандидат технических наук, проректор по учебной работе, заведующий кафедрой электрических станций, подстанций и диагностики электрооборудования, Россия, Иваново, e-mail: avgus@ispu.ru

\section{Андрей Александрович Дьячков}

ФГБОУВО «Ивановский государственный энергетический университет имени В.И. Ленина», учебный мастер кафредры высоковольтной электроэнергетики, электротехники и электрофизики, Россия, Иваново, телефон (4932) 26-97-23

\section{Владимир Дмитриевич Лебедев}

ФГБОУВО «Ивановский государственный энергетический университет имени В.И. Ленина», кандидат технических наук, заведующий кафедрой автоматического управления электроэнергетическими системами, Россия, Иваново, e-mail: vd_lebedev@mail.ru

\section{Александр Михайлович Соколов}

ФГБОУВО «Ивановский государственный энергетический университет имени В.И. Ленина», доктор технических наук, доцент кафедры высоковольтной электроэнергетики, электротехники и электрофизики, Россия, Иваново, телефон (4932) 26-99-04, e-mail: alex2010fn@yandex.ru

\section{Тимофей Евгеньевич Шадриков}

ФГБОУВО «Ивановский государственный энергетический университет имени В.И. Ленина», кандидат технических наук, доцент кафедры высоковольтной электроэнергетики, электротехники и электрофизики, Россия, Иваново, телефон (4932) 26-99-04, e-mail: Pr3d37@gmail.com

\section{Комплексная методика расчета несинусоидальных систем переменного тока повышенной частоты}

\section{Авторское резюме}

Состояние вопроса. При исследовании и разработке принципиально новых электротехнических комплексов повышенной частоты выявлены проблемы с расчетами и моделированием режимов работы таких систем по методике Ю.М. Осипова. Применение методики дает результаты, значительно отличающиеся от экспериментальных. Целью исследования является совершенствование методики в направлении учета особенностей электротехнических комплексов повышенной частоты, их топологии и компонентов.

Материалы и методы. Исследование проведено на математической модели электротехнического комплекса повышенной частоты, включающей в себя источник питания, согласующие трансформаторы, кабельные линии и нагрузку. Источник питания моделируется в упрощенной фрорме с наличием внутреннего сопротивления транзисторов.

Результаты. Разработана аналитическая методика расчета и моделирования систем переменного тока повышенной частоты. Выведены аналитические выражения для расчета характеристик и параметров компонентов электротехнических комплексов повышенной частоты. Даны рекомендации по автоматизации разработанной методики с применением MATLAB, Python или Simulink. Выполнено сравнение результатов расчета и эксперимента в целях подтверждения их достоверности.

Выводы. Выполненные разработки могут быть использованы для моделирования и расчета систем переменного тока повышенной частоты в целях определения токовой загрузки, уровней напряжения, анализа перенапряжений и аварийных режимов.

Ключевые слова: частотный анализ, инвертор, двухпроводный кабель, напряжение повышенной частоты, преобразования Фурье, трансформаторы повышенной частоты, электротепловая обработка, электромобили

\section{Alexey Vasilievich Gusenkov}

Ivanovo State Power Engineering University, Candidate of Engineering Sciences, Academic Vice-Rector, Head of Electric Power Plants, Substations and Electrical Equipment Diagnostics Department, Russia, Ivanovo,

e-mail: avgus@ispu.ru

() Гусенков А.В., Дьячков А.А., Лебедев В.Д., Соколов А.М., Шадриков Т.Е., 2021

Вестник ИГЭУ, 2021, вып. 2, с. 40-54. 
Andrey Alexandrovich Dyachkov

Ivanovo State Power Engineering University, Training Master of High-Voltage Electric Power Engineering, Electrical Engineering and Electrophysics Department, Russia, Ivanovo, telephone: (4932) 26-97-23

\section{Vladimir Dmitrievich Lebedev}

Ivanovo State Power Engineering University, Candidate of Engineering Sciences, Head of Automatic Control of Electric Power Systems Department, Russia, Ivanovo, e-mail:vd_lebedev@mail.ru

\section{Alexander Mikhailovich Sokolov}

Ivanovo State Power Engineering University, Doctor of Engineering Sciences, Associate Professor of High-Voltage Electric Power Engineering, Electrical Engineering and Electrophysics Department, Russia, Ivanovo, telephone: (4932) 26-99-04,

e-mail: alex2010fn@yandex.ru

\section{Timofey Evgenievich Shadrikov}

Ivanovo State Power Engineering University, Candidate of Engineering Sciences, Associate Professor of High-Voltage Electric Power Engineering, Electrical Engineering and Electrophysics Department, Russia, Ivanovo, telephone: (4932) 26-99-04, e-mail: Pr3d37@gmail.com

\section{Integrated methodology to calculate nonsinusoidal AC systems of high frequency}

\section{Abstract}

Background. During the research and development of fundamentally new AC systems of high frequency, the authors identified the problems of the calculations and modeling of the operating modes of such systems according to the method of Yu.M. Osipova. Application of the method gives results that are significantly different from the experimental ones. The aim of the study is to improve the methodology considering the features of high-frequency electrical systems, their topology, and components.

Materials and methods. The research has been carried out based on a mathematical model of an electrical system of high frequency, which includes a power supply, matching transformers, cable lines and a load. The power supply is modeled in a simplified form with the internal resistance of the transistors.

Results. An analytical technique for calculating and modeling high-frequency AC systems has been developed. Analytical expressions are presented to calculate the characteristics and parameters of the high frequency system components. Recommendations on automation of the developed technique using MATLAB, Python or Simulink are presented.Comparison of the results of calculation and the experiment is carried out to confirm the reliability.

Conclusions. The results of the research work can be used for modeling and calculating high frequency alternating current systems to determine the current load, voltage levels, analyze overvoltages and emergency modes.

Key words: frequency analysis, inverter, two-wire cable, high frequency, Fourier transform, high frequency transformers, electrothermal treatment, electric vehicles

DOI: 10.17588/2072-2672.2021.2.040-054

Введение. Разработка и применения систем переменного тока повышенной частоты с различной фрормой выходного напряжения в настоящее время является актуальной задачей. В основе таких систем лежит использование преобразователей напряжения (voltage source converter (inverter) VSC/VSI), выполненных на мощных полупроводниковых элементах - IGBTтранзисторах. Подобный подход лег в основу систем постоянного тока высокого напряжения (VSC HDVC).

В отличие от HVDC, системы переменного тока повышенной частоты и их компоненты имеют более широкие перспективы применения, в том числе вне большой энергетики. К их числу можно отнести разработку и применение синхронных генераторов повышенной частоты с преобразователями напряжения [1-3], разработку блоков ПГУ с генераторами, производящими напряжение повышенной частоты [2-5], разработку зарядной инфраструктуры и сети зарядных станций для электробусов и электромобилей $[6,7]$, обработку композиционных материалов токами повышенной частоты [8, 9] систем освещения и др.

Опыт практического применения ЭТКПЧ показал [8], что подобные комплексы могут успешно применяться для целей термической (электротепловой) обработки 
различных композиционных материалов и индукционного нагрева. В ходе НИОКР была выявлена острая необходимость в создании расчетной методики и математической модели, позволяющих описать ЭТКПЧ различной конфигурации. Необходимость разработки расчетной методики и модели продиктована широким использованием инструментов автоматизации с применением современного ПО (например, MATLAB, Simulink).

Ранее [10, 11] были выполнены исследования в разработке методов расчета и моделирования систем переменного тока повышенной частоты. Первоначально на основе частотного анализа [10] была разработана методика расчета электрических цепей, содержащих транзисторные преобразователи напряжения, позволяющая использовать метод частотного анализа на основе разложения воздействующего напряжения в ряд Фурье.

В дальнейших исследованиях было произведено совершенствование методики [11] в целях оптимизации процедуры вычислений на основе численного интегрирования и уточнения параметров расчета внутреннего сопротивления источника питания, выполненного на IGBTтранзисторах [12].

Ниже приводится обобщенная методика расчета несинусоидальных систем переменного тока повышенной частоты в установившемся режиме работы.

Методика включает в себя следующие этапы:

- составление и анализ схемы замещения сетей переменного тока повышенной частоты;

- определение параметров схемы замещения (в том числе, компонентов ЭТКПЧ);

- определение частотных эффектов и их влияния на характеристики компонентов (транзисторов, трансформаторов, кабелей и т.п.);

- определение особенностей применения частотного анализа для расчета установившихся режимов работы таких систем.

По результатам применения методики к конкретному комплексу повышенной частоты может быть построена математическая модель, пригодная для выполнения следующих процедур:

- расчета токов и напряжений в узлах системы;
- определения минимальной и максимальной токовой нагрузки (режимов работы) и выбора соответствующих компонентов;

- анализа перенапряжений, резонансных эффректов и предельно допустимых режимов работы изоляции;

- расчета и анализа режимов холостого хода и короткого замыкания;

- гармонического анализа физических параметров (мощности, тока, напряжения);

- определения потерь мощности, КПД и анализа эфффективности.

Методика рассматривается применительно к варианту ЭТКПЧ магистрального типа и единичному устройству электротепловой обработки (ЭТО), как пример ЭТКПЧ в частной реализации.

Схема замещения. Для исследования режимов работы и анализа процессов, происходящих в ЭТКПЧ, необходимо правильно составить эквивалентную схему замещения с учетом конфигурации ЭТКПЧ и его отличительных особенностей.

В зависимости от способа применения комплекса повышенной частоты, были разработаны несколько его конфигураций:

- единичное устройство повышенной частоты (например, электротепловая обработка одного или нескольких конкретных изделий из композита на предприятиях малого бизнеса) (рис. 1,a);

- ЭТКПЧ радиального типа, предназначенный для организации электропитания с использованием кабельных линий высокого напряжения и значительного числа потребителей в виде объектов электротепловой обработки, подключенных на конце кабельной линии (рис. 1,б);

- ЭТКПЧ магистрального типа, предназначенный для организации электропитания с использованием кабельных линий высокого напряжения и наличием потребителей как в конце кабельной линии, так и в промежуточных точках (присоединения) (рис. 1,б).

Единичное устройство повышенной частоты для электротепловой обработки состоит из преобразователя на основе биполярных транзисторов и согласующего трансформатора повышенной частоты. Эквивалентная схема замещения (на рис. 1,a) показана на рис. 2. 


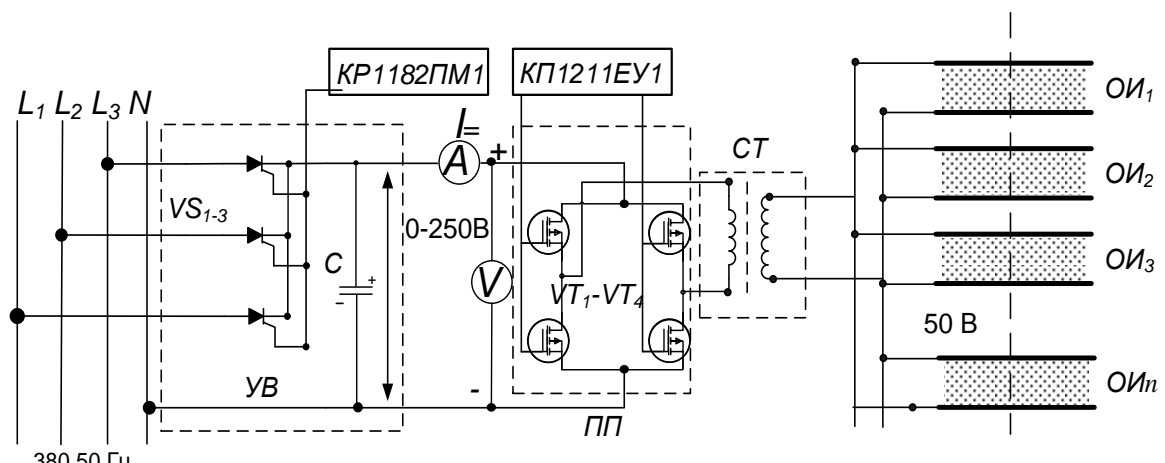

a)

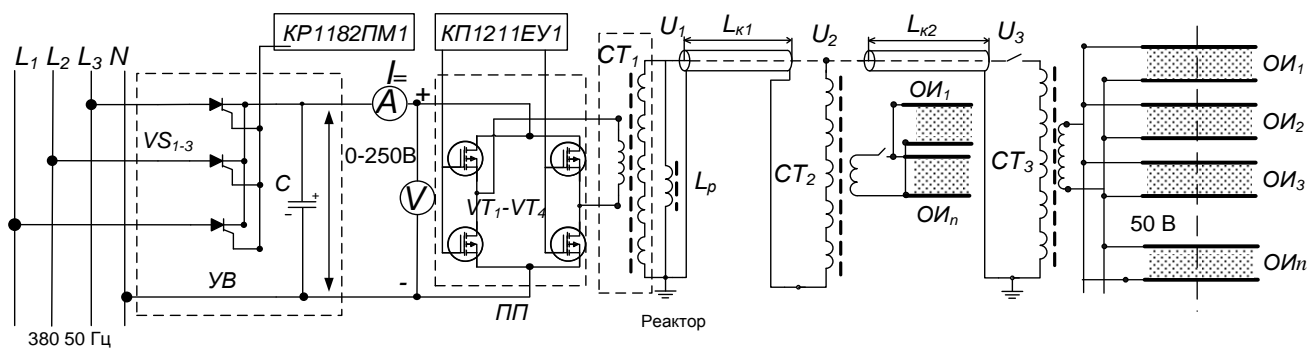

б)

Рис. 1. Принципиальная схема устройства электротепловой обработки (а) и электротехнического комплекса повышенной частоты с различной конфигурацией (б): УВ - управляемый выпрямитель; ПП полупроводниковый преобразователь; СТ - согласующий трансформатор; ОИ - обрабатываемое изделие; $\mathrm{C}$ - емкость на шинах постоянного тока; $\mathrm{VT}_{1}-\mathrm{VT}_{4}, \mathrm{VD}_{1}-\mathrm{VD}_{4}-$ силовые транзисторы и диоды полупроводникового преобразователя; $I_{k}$ - кабельная линии электропередачи; $L_{p}$ - реактор

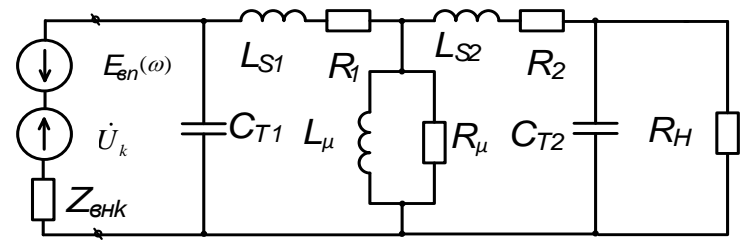

Рис. 2. Схема замещения ЭТКПЧ (рис. 1,a): $U_{k}$ - источник напряжения $k$-й гармоники, иллюстрирующий работу преобразователя напряжения; $E_{\text {вп }}$ - противо-ЭДС; $Z_{\text {внк }}$ - внутреннее сопротивление преобразователя напряжения, необходимое для учета зависимости сопротивления транзистора в открытом состоянии от частоты (будет показано далее); $C_{T 1}$ и $C_{T 2}$ - емкость обмоток низшего и высшего напряжения силового транссоорматора; $L_{s 1}$ n $L_{s 2}$ - индуктивности рассеивания обмоток низшего и высшего напряжения силового трансформатора; $R_{1}$ и $R_{2}$ - активные сопротивления обмоток низшего и высшего напряжения силового трансформатора; $R_{\mathrm{H}}$ - активное сопротивление нагрузки; $R_{\mu}$ и $L_{\mu}$ - активное сопротивление и индуктивность ветви намагничивания трансформатора
При составлении общей эквивалентной схемы замещения ЭТКПЧ может потребоваться использование эквивалентных схем замещения следующих компонентов $[12,13]:$

- источника питания повышенной частоты;

- повышающих/понижающих трансформаторов;

- высоковольтной кабельной линии;

- нагрузки (как правило, носит чисто активный или активно-индуктивный характер).

В случае, если осуществляется централизованное электропитание множества точек ЭТО, необходимо использовать иной порядок составления схемы замещения (рис. 1,б). Необходимо также учитывать различия конфигураций радиального и магистрального комплексов (рис. 1).

ЭТКПЧ, представленный на рис. 1,б, имеет в своем составе радиальную высоковольтную линию электроснабжения повышенной частоты с питанием от транзисторного преобразователя. Радиальная схема предполагает наличие в этой конфигурации одной включенной между источни- 
ком питания и электроприемником без дополнительных присоединений в промежуточных точках высоковольтной кабельной линии. Такой вариант ЭТКПЧ является упрощенным вариантом ЭТКПЧ магистрального типа в случае наличия одного потребителя на конце линии.

ЭТКПЧ магистрального типа основан на применении высоковольтной кабельной линии с наличием промежуточных точек подключения устройств электротепловой обработки (в общем случае число устройств ЭТО, а значит, и число отпаек, может составлять $i$ единиц). Схема замещения ЭТКПЧ магистрального типа приведена на рис. 3.

По аналогии с составлением схемы замещения на рис. 3 могут быть рассмотрены любые конфигурации несинусоидальных электрических сетей с произвольной величиной частоты питающей ЭДС.

Определение параметров и характеристик схемы замещения. Определение параметров схемы замещения ЭТКПЧ необходимо для анализа установившихся и переходных режимов его работы (например, с применением метода частотного анализа $[10,11])$. Разработанные методики определения параметров схем замещения компонентов ЭКТПЧ могут быть использованы, в том числе, для проведения верификации экспериментальных и теоретических исследований [10, 11].

Определение параметров преобразователя напряжения (инвертора). Основным компонентом источника питания в ЭТКПЧ является полупроводниковый преобразователь напряжения (инвертор, построенный по мостовой или полумостовой схеме). В его основе лежит мостовая сборка управляемых IGBT-транзисторов, система управления которых построена на задающем генераторе или драйвере. В схеме замещения на рис. 3 источник питания (инвертор) замещается противо-ЭДС, источником напряжения и комплексным сопротивлением $Z_{\text {внк. }}$

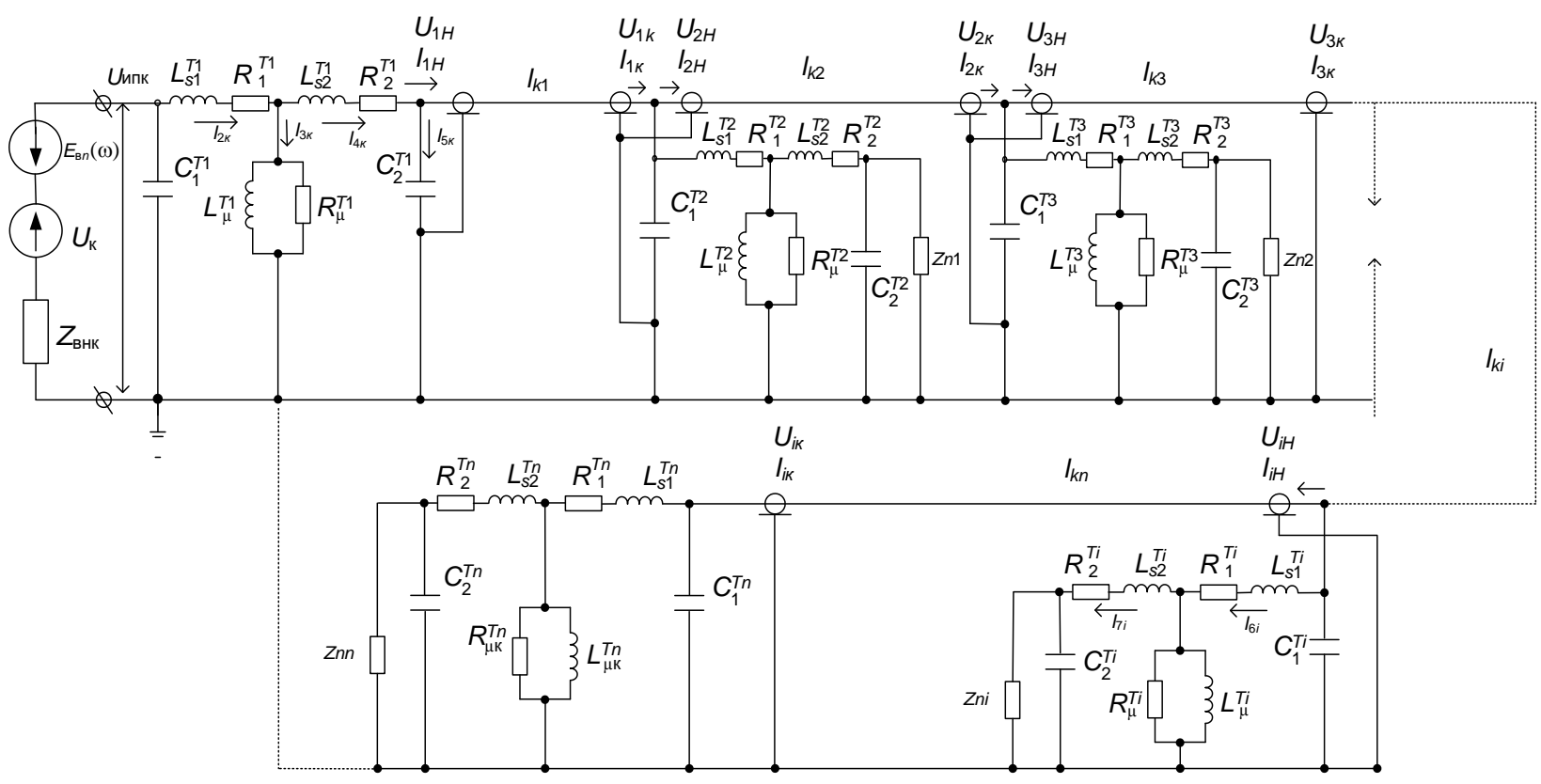

Рис. 3. Схема замещения ЭТКПЧ магистрального типа: $R_{1}^{T n}$ и $R_{2}^{T n}$ - активные сопротивления обмоток высшего и низшего напряжения трансформатора $T_{P . N} ; L_{s 1}^{T n}$ и $L_{s 2}^{T n}-$ индуктивности рассеяния этих обмоток; $R_{\mu k}^{T n}$ - величина активного сопротивления, отражающего потери мощности в магнитопроводе силового трансформатора $T_{N}$ для $k$-й гармоники; $L_{\mu k}^{T n}$ - индуктивность намагничивания магнитопровода для $k$-й гармоники этого трансформатора; $R_{\mu k}^{T n}$ и $L_{\mu k}^{T n}$ - вычисляются по соответствующей методике [13-15]; $C_{1}^{T n}$ и $C_{2}^{T n}$ - емкости первичной и вторичной обмоток трансформатора $T_{P . N}$ относительно земли 
Проведенные ранее исследования $[16,17,21]$ показали наличие фракта запаздывания появления тока коллектора относительно тока эмиттера в транзисторе и позволили получить основополагающее выражение для определения внутреннего сопротивления транзистора от кратности гармоники:

$Z_{\text {вн }}=\frac{Z_{\text {вно }}}{|\alpha(\omega)|}=Z_{\text {вно }} \sqrt{1+\left(\frac{k f_{1}}{f_{\mathrm{a}}} m\right)^{2}}$,

где $Z_{\text {вно }}=\frac{\Delta U_{T}}{I_{k 0}}-$ внутреннее сопротивление транзистора в открытом состояния при постоянном напряжения; $k-1,3,5 \ldots$ кратность гармоники (в кривой ЭДС присутствуют только нечетные гармоники [21]); $f_{1}$ - частота первой гармоники; $f_{\text {a }}$ - предельная частота усиления (паспортное значение); $|\alpha(\omega)|$ - модуль коэфффициента передачи транзистора по току; $\Delta U_{T}$ - падение напряжения на открытом транзисторе при постоянном токе $I_{\text {ko }}$ (паспортные данные транзистора); $m$ - поправочный коэфффициент безразмерного характера.

В [11] показано, что для выполнения расчетов с достаточной сходимостью можно использовать величину $m=\sqrt{3}$.

В (1) используется следующее выражение для коэффрициента передачи по току $\alpha(\omega)$ :

$$
|\alpha(\omega)|=\frac{\alpha_{0}}{\sqrt{1+\left(\frac{\omega}{\omega_{a}} m\right)^{2}}}=\frac{\alpha_{0}}{\sqrt{1+\left(\frac{k f_{1}}{f_{a}} m\right)^{2}}},
$$

где $\alpha_{0} \approx 1$ - коэффрициент передачи по току на постоянном напряжении; $f$ - произвольное значение частоты; $\omega=2 \pi f ; \omega_{a}=2 \pi f_{\text {a }}$.

Путем математических преобразований и умножения (1) на ток коллектора получаем

$\Delta U_{T}(\omega)=\Delta U_{\mathrm{T}}\left(1+j \frac{k f_{1}}{f_{\mathrm{a}}}\right)+E_{\mathrm{BH}}(\omega)$,

где $\Delta U_{T}(\omega)$ - падение напряжения на открытом транзисторе при произвольных значениях частоты напряжения и кратности гармоники; $E_{\mathrm{B}}(\omega)$ - противо-ЭДС, отражающее скорректированное значение внутреннего сопротивления (1):

$$
E_{\mathrm{B \Pi}}(\omega)=j l_{\mathrm{к} 0} \cdot Z_{\mathrm{вн}} \frac{k f_{1}}{f_{\mathrm{a}}}=j \Delta U_{\mathrm{T}} \frac{k f_{1}}{f_{\mathrm{a}}} .
$$

Выражения (3) и (4) позволяют предложить новый вариант схемы замещения инвертора, представленный рис. 2, 3, в том случае, если для определения внутреннего сопротивления используется выражение (1).

В случае, если внутреннее сопротивление транзистора определяется с помощью формулы (1), в схеме замещения инвертора необходимо исключить противо-ЭДС.

Определение параметров силового трансформатора повышенной частоты. Применение трансформаторов повышенной частоты в составе ЭТКПЧ обусловлено, с одной стороны, необходимостью повышения/понижения величины рабочего напряжения, с другой стороны, необходимостью обеспечения безопасной величины напряжения на нагрузке согласно требованиям ПУЭ ${ }^{5}$.

Ниже приводится способ определения параметров схемы замещения повышающего силового трансформатора повышенной частоты. Базовые основы расчета и проектирования импульсных и силовых трансформаторов повышенной частоты приведены в $[13,14]$.

Такой трансформатор повышенной частоты обозначен на рис. 3 как $T_{1}$. Конструкция трансформатора представлена на рис. 4. В целях уменьшения емкости трансформатора обмотка высшего напряжения выполнена секциями многослойного типа (рис. 4).

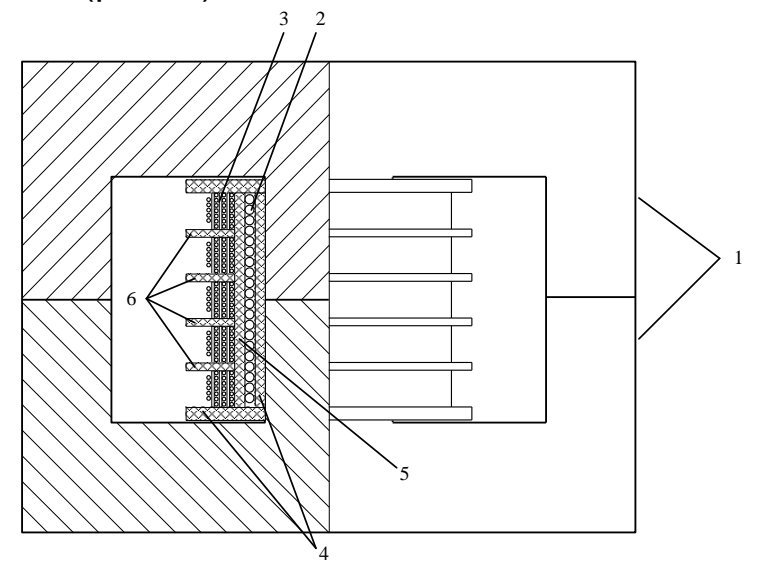

Рис. 4. Конструкция высоковольтного силового трансформатора повышенной частоты: 1 - ферритовый магнитопровод; 2 - обмотка $\mathrm{HH}$; 3 - обмотка ВН; 4 - каркас; 5 - изоляция между обмотками ВН и НH; 6 - секционные прокладки обмотки $\mathrm{BH}$

5 Правила устройства электроустановок (ПУЭ). 7-е изд. [Утверждены приказом Минэнерго Российской Федерации от 08.07.2002. № 204]. - М.: Омега-Л, 2012. - 272 с. 
Для определения параметров схемы замещения трансформатора необходимо рассмотреть конструкцию его обмотки согласно методике [13].

В случае многослойной обмотки высшего напряжения конструкция трансформатора представлена на рис. 5 .

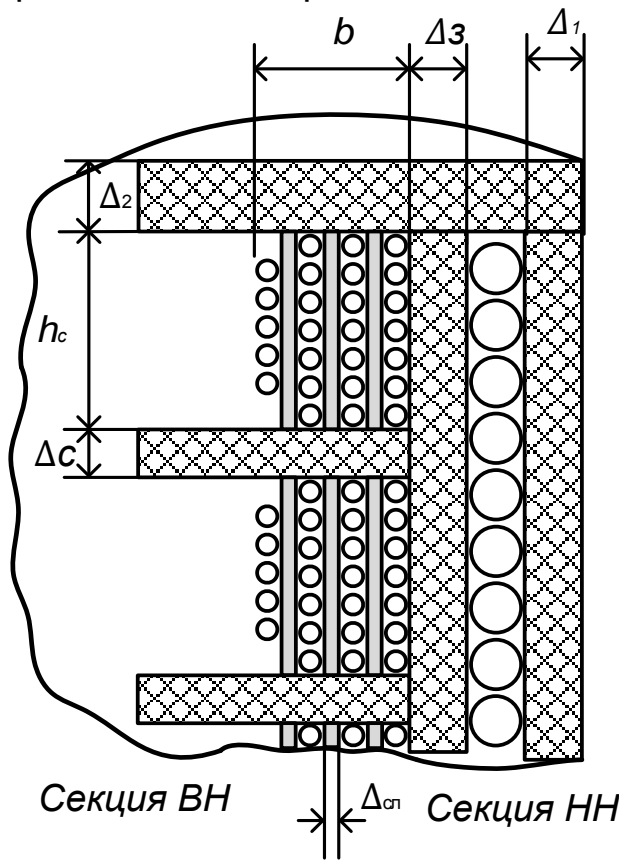

Рис. 5. Чертеж обмоток силового трансформатора повышенной частоты: ВН и НH - секции обмоток высшего и низшего напряжения трансформатора

Емкость обмотки низшего напряжения составляет

$$
C_{1}=\frac{\varepsilon \varepsilon_{0} l_{\mathrm{CPB}}\left(h-2 \Delta_{2}\right)}{3 \Delta_{1}},
$$

где $\varepsilon-$ диэлектрическая проницаемость материала изоляции; $\varepsilon_{0}$ - электрическая постоянная; $h$ - высота секции в сечении; $I_{\text {СРв }}$ средняя длина витка (определяется с учетом типоразмера сердечника, каркаса и т.п.); $\Delta_{3}-$ толщина изоляции между низковольтной и высоковольтной обмотками; $\Delta_{1}$ и $\Delta_{2}$ - толщины, определяемые конструктивным исполнением изоляционных прокладок (рис. 5).

Емкость обмотки высокого напряжения в общем случае зависит от числа секций $(m)$ в составе обмотки. Вывод выражения для определения емкости $C_{2}$ обмотки высшего напряжения, состоящей из 5 секций (рис. 4), приведен в [13, 14].

Индуктивности рассеивания обмоток рассчитываются по формуле

$$
L_{S 1}=\frac{\mu_{0} w_{1}^{2} I_{\mathrm{CPB}}}{n_{\mathrm{CO}} h+\Delta_{\mathrm{c}}\left(n_{\mathrm{C}}-1\right)}\left(\Delta_{3}+\frac{d_{1}+d_{2}}{3}\right),
$$

где $w_{1}$ - число витков первичной обмотки; $h$ - высота секции в сечении (рис. 5); $d_{1}-$ диаметр (высота) провода первичной обмотки; $d_{2}$ - диаметр (высота) провода вторичной обмотки; $n_{\mathrm{co}}-$ число секций обмотки высшего напряжения.

Индуктивность высоковольтной обмотки определяется суммированием индуктивностей рассеяния всех секций обмотки:

$$
\begin{aligned}
& L_{S B}=\frac{\mu_{0} w_{2}^{2} I_{\mathrm{CPB}} n_{\mathrm{CO}}}{h}\left(\Delta_{3}+\right. \\
& +\frac{1}{m^{2}} \sum_{k=1}^{m-1}\left(\Delta_{\mathrm{C} Л \mathrm{~T}}(m-k)^{2}+\frac{d_{1}}{3}+\right. \\
& \left.+\frac{1}{3} \sum_{k=0}^{m-1} d_{2} F(k, m)\right),
\end{aligned}
$$

где $m$ - число слоев обмотки в одной секции; $F(k, m)$ - функция числа слоев обмотки, определяемая по выражению

$$
F(k, m)=3\left[\left(1-\frac{k}{m}\right)\left(1-\frac{k+1}{m}\right)+\frac{1}{m^{2}}\right] .
$$

Индуктивность намагничивания определяется следующим выражением [13, 14]:

$L_{\mu}=\frac{\mu \mu_{0} w_{1}^{2} S_{\mathrm{C}} k_{\mathrm{a}}}{l_{\mu}}$,

где $\mu$ - относительная магнитная проницаемость магнитного материала магнитопровода (для фрерритовых сердечников $\mu=1700-1800) ; K_{\mathrm{a}}-\leq 1-$ коэфрфициент заполнения сечения магнитопровода (для ферритовых сердечников $\left.K_{\mathrm{a}}=1\right) ; l_{\mu}$ - длина средней силовой линии магнитного поля в магнитопроводе.

Активные сопротивления первичной и вторичной обмоток определяются по выражению $[13,14]$

$$
R_{1,2}=\frac{4 I_{\mathrm{CPB}} w_{1,2} \rho_{\mathrm{M}}}{n_{\text {пр }} \pi d_{\Pi \mathrm{P}}^{2}},
$$

где $n_{\text {пр }}$ - число одинаковых, параллельно включенных проводов; $d_{\text {пр }}$ - диаметр проводников; $\rho_{\text {м }}$ - удельное сопротивление материала проводника (меди); $w$ - число витков обмотки ВН или НН соответственно.

Величина активного сопротивления, отражающего потери в магнитопроводе силового трансформатора, определяется по фрормуле

$$
R_{\mu}=\frac{U_{k}^{2}}{1,64 \cdot 10^{-3} \cdot f_{k}^{1,31} B_{k}^{2,49} \delta V},
$$

где $\delta, V$ - плотность материала сердечника и объем магнитопровода соответственно; $B_{k}-$ индукция магнитного поля в маг- 
нитопроводе для $k$-й гармоники; $f_{k}-$ частота $k$-й гармоники.

Указанные выше выражения могут быть применены и для других вариантов конструктивного исполнения трансформаторов повышенной частоты лишь с небольшими изменениями, используя способ и методику, изложенную в [13].

Определение параметров кабельных линий. Представленные на рис. 1,а и 2 схемы замещения единичного устройства электротепловой обработки не предполагают наличия в его составе кабельных линий повышенной частоты. В свою очередь, при реализации радиального или магистрального вариантов ЭТКПЧ для передачи и распределения энергии могут быть использованы кабельные линии высокого напряжения повышенной частоты.

Первоначально в методике, предложенной в $[10,11]$, рассматривалась возможность применения П-образной схемы замещения с сосредоточенными параметрами для моделирования кабельной линии повышенной частоты. Такое допущение возможно при длине кабельной линии не более 15-20 м, однако для расчета радиальных и магистральных ЭТКПЧ целесообразно использовать уравнения длинной линии $[25,26]$.

При выполнении расчетов для схемы замещения ЭТКПЧ магистрального типа (рис. 3), когда линия представлена объектом с распределенными параметрами, для описания этого элемента необходимо использовать уравнения длинной линии:

$\dot{U}_{1}=\dot{U}_{2} \operatorname{ch} \gamma I_{K}+\dot{I}_{2} z \cdot \operatorname{sh} \gamma I_{K}$;

$i_{1}=\dot{I}_{2} \operatorname{ch} \gamma I_{K}+\frac{\dot{U}_{2}}{z} \operatorname{sh} \gamma I_{K}$;

$\dot{U}_{2}=\dot{U}_{1} \operatorname{ch} \gamma I_{K}-\dot{I}_{1} z \cdot \operatorname{sh} \gamma I_{K}$

$\dot{I}_{2}=\dot{I}_{1} \operatorname{ch} \gamma I_{K}-\frac{\dot{U}_{1}}{z} \operatorname{sh} \gamma I_{K}$,

где $\dot{U}_{1}, \dot{i}_{1}$ и $\dot{U}_{2}, \dot{i}_{2}$ - значения напряжения и тока одной и той же гармоники в начале и в конце линии соответственно в комплексной форме; $z$ - волновое сопротивление, определяемое по выражению

$z=\sqrt{\frac{L_{0}}{C_{0}}} ;$

$\gamma$ - коэффрициент распространения волны в линии, определяемый по фрормуле $\gamma=\alpha+j \beta$,

где $\alpha$ - коэфффициент затухания волны в линии:

$$
\alpha=2 \pi f_{k} \sqrt{L_{0} C_{0}} \cdot \sqrt{\frac{1}{2}\left[\sqrt{1+\left(\frac{R_{0}}{2 \pi f_{k} L_{0}}\right)^{2}}-1\right]} ;
$$

$\beta$ - коэфффициент фразы:

$$
\beta=2 \pi f_{k} \sqrt{L_{0} C_{0}} \cdot \sqrt{\frac{1}{2}\left[\sqrt{1+\left(\frac{R_{0}}{2 \pi f_{k} L_{0}}\right)^{2}}+1\right]},
$$

где $R_{0}, L_{0}$ и $C_{0}$ - погонные параметры линии: активное сопротивление, индуктивность и емкость изоляции

В случае применения кабелей коаксиальной конструкции (например, РК-50, РК-75) погонные реактивные характеристики $\left(L_{0}\right.$ и $\left.C_{0}\right)$ могут быть определены посредством справочных (паспортных) данных, с применением эксперимента либо по методике, приведенной в [23].

Для снижения реактивной мощности в системах переменного тока повышенной частоты необходимо рассмотреть возможность внедрения двухпроводных кабельных линий с общим экраном (рис. 6). Такие кабельные линии могут быть выполнены с литой (твердой) или воздушной изоляцией, обладают большим волновым сопротивлением и меньшей погонной емкостью, что благоприятно сказывается на режимах работы ЭТКПЧ [22].

В случае использования такой линии для реализации управляемой электропередачи и с учетом фазового сдвига 180 градусов между напряжениями $U_{1}$ и $U_{2}$ погонную емкость можно определить с помощью следующего выражения [23-26]:

$$
C_{0}=\frac{\pi \varepsilon}{\ln \left(\frac{R_{\ni}^{2}-d^{2}}{R_{\ni}^{2}+d^{2}} \cdot \frac{2 d}{r}\right)} .
$$

Наиболее сложным вариантом определения параметров кабельной линии является случай, когда проводники двухпроводной линии имеют твердую изоляцию и расположены в заполненной воздухом проводящей оболочке (рис. 6,б). Дополнительно возможны случаи, когда проводники расположены прямо на экране (частный случай варианта схемы 6,б). Вывод фрормул погонных параметров с использованием потенциальных коэффрициентов для таких случаев представлен в [27]. 


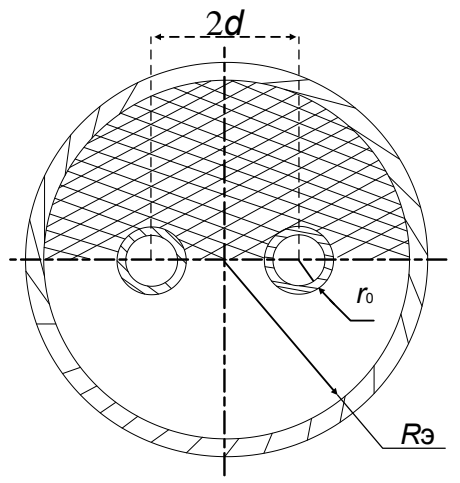

a)

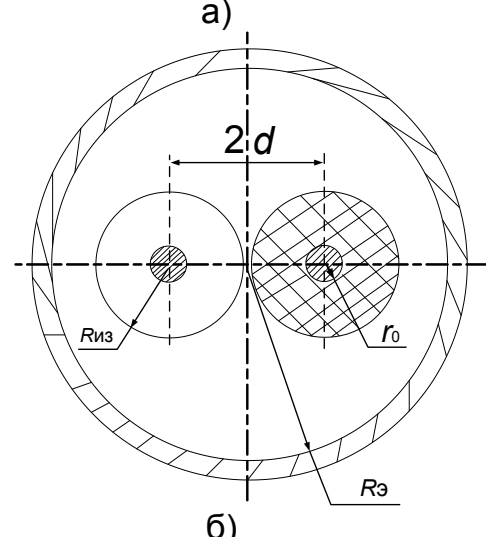

б)

Рис. 6. Варианты двухпроводной кабельной линии

Методика частотного анализа. Для электрических цепей при воздействии синусоидальных периодических величин, как правило, используют комплексную фрорму записи. Для достижения максимальной передаваемой мощности в нагрузку в ЭКТПЧ на выходе инвертора могут быть использованы различные формы воздействующей ЭДС, например прямоугольная форма, трапеция, трапеция с паузой. Последние две получаются естественным образом путем сглаживания прямоугольной фрормы при работе на индуктивную и емкостную нагрузку. Наличие паузы благоприятно сказывается на работе силовых транзисторов. Таким образом, фоорма напряжения «трапеция с паузой» является основной в ЭТКПЧ $[10,11]$. Указанные формы воздействующих ЭДС полупроводникового преобразователя в ЭКТПЧ представлены на рис. 7.

При периодических несинусоидальных воздействиях необходимо использовать метод частотного анализа, т.е. выполнить разложение воздействующего на электрическую цепь напряжения (ЭДС) в ряд Фурье [23, 24].

Прямоугольная и трапецеидальная форма ЭДС имеют готовые выражения для разложения в ряд Фурье [23], для тра- пеции с паузой можно воспользоваться наработками в [24].
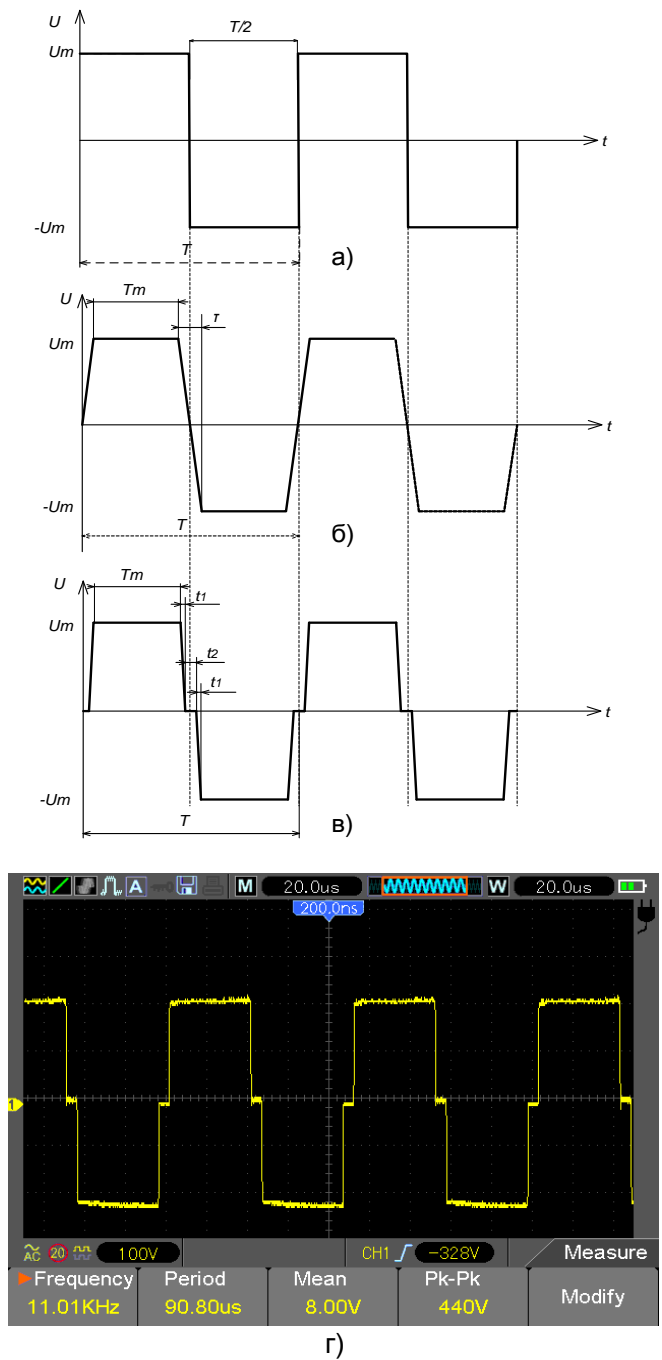

Рис. 7. Формы воздействующих ЭДС в ЭТКПЧ: а-в - теоретические представления форм; г реальная осциллограмма ЭДС в ЭКТПЧ

Напряжение (рис. 7,г), получаемое с выхода транзисторного преобразователя (рис. 1, 2, 3) и подаваемое в кабельную линию, имеет разложение в ряд Фурье $[22,27]$ : $U(t)=\frac{4 U_{m}}{\pi} \sum_{k=1}^{\infty} \frac{\sin \left(k \omega_{1} t\right)}{k}=$

$=\sum_{k=1}^{\infty} U_{k} \sin \left(\omega_{k} t\right)=\sum_{k=1}^{\infty} U_{k}(t)$,

где $U_{k}=\left(4 U_{k}\right) / \pi k-$ амплитуда $k$-й гармоники; $f_{k}=k f_{1}=k / T_{1}$ и $\omega_{k}=2 \pi f_{k}-$ частота и угловая частота $k$-й гармоники; $k=1,3,5,7 \ldots$ кратность гармоники (присутствуют только нечетные гармоники).

Кривую на рис. 7,в можно рассматривать как функцию, имеющую точки разрыва и разбитую на участки (рис. 8). 


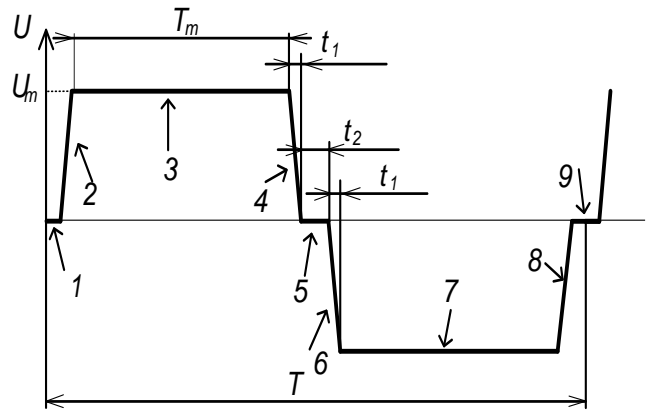

Рис. 8. Иллюстрация представления кривой ЭДС, разбитой на участки для выполнения интегрирования

Интеграл от такой функции определяется как сумма интегралов в пределах тех участков, где она остается непрерывной и может быть описана аналитически (рис. 8) $[24,25]$. Кривую «трапеция с паузой» (рис. 7,в) можно представить в нескольких участках (рис. 8): нулевое значение, рост, время максимального значения, спад, нулевое значение и т.д.

$U_{k}=\frac{2}{T} \int_{0}^{\mathrm{T}} U(t) \cdot \sin \left(k \omega_{1} t\right) d t=2 U_{m} f_{1} \sum_{i=1}^{n} I_{n}$.

Интеграл за один период изменения ЭДС (17) во времени необходимо представить как сумму всех слагаемых, каждое из которых соответствует одному из этих участков, полагая, что в течение паузы (рис. 7,В,г, рис. 8) значение ЭДС составляет $U(t)=0$, на участках амплитуды $U(t)=U_{m}$ и $U(t)=-U_{m}$ соответственно, а на участках роста/спада имеет место изменение напряжения по линейному закону.

Полученные в [11] выражения позволяют при определении амплитуды гармоник ЭДС с помощью формулы (17) не выполнять численное интегрирование и ускорить вычисления при расчете установившихся режимов с помощью аналитических выражений.

При использовании частотного анализа для расчета установившегося режима ЭКТПЧ обязательно необходимо принять во внимание наличие поверхностного эффекта в проводниках обмоток силового трансформатора и жилах кабельной линии. Явление поверхностного эфффекта оценивается величиной эквивалентной глубины проникновения электромагнитной волны в проводник при протекании по нему переменного тока (в случае ЭКТПЧ тока повышенной частоты). Величина эквивалентной глубины проник- новения электромагнитной волны оценивается с помощью выражения

$$
b_{m}=\sqrt{\frac{\rho_{M}}{\pi k f_{1} \mu_{0}}} \text {. }
$$

При этом должны быть скорректированы величины активных сопротивлений обмоток силового трансформатора и жилы кабельной линии (10) с учетом воздействующей частоты и глубины проникновения волны в проводнике:

$$
\left\{\begin{array}{l}
R_{\text {Т(л) }}=\frac{4 \rho_{\mathrm{M}} I}{N \pi d^{2}} \text { при } b_{m} \geq \frac{d}{2}, \\
R_{\text {Т(Л) }}=\frac{l \rho_{M}}{N \pi\left(d b_{m}-b_{m}^{2}\right)} \text { при } b_{m}<\frac{d}{2} .
\end{array}\right.
$$

Дополнительно необходимо отметить, что выражения (1),(4),(11),(14) зависят от частоты гармоники, поэтому для корректной реализации метода частотного анализа расчет каждого параметра схемы замещения на каждой итерации должен учитывать влияние частоты.

Сопротивления остальных реактивных элементов схемы замещения (рис. 3) определяются как

$$
\begin{aligned}
& j X_{L i}=j \omega_{k} L_{i}=j 2 \pi k f_{1} L_{i}, \\
& j X_{C i}=\frac{1}{j \omega_{k} C_{i}}=\frac{1}{j 2 \pi k f_{1} C_{i}} .
\end{aligned}
$$

Автоматизация методики расчета установившихся режимов ЭТПКЧ с помощью частотного анализа. Для выполнения автоматизации расчета установившихся режимов ЭТКПЧ могут быть применены MATLAB и Simulink.

При алгоритмизации в MATLAB выполняется вычисление амплитуды гармоник разложения Фурье для заданного варианта осциллограммы ЭДС (рис. 7) с увеличением времени начиная от нулевого значения. В каждый момент времени производится расчет токов и напряжений схемы замещения (рис. 3) ЭТКПЧ в комплексной фрорме для всех гармоник разложения [22]. С учетом модификации методики расчета [11] в настоящее время возможно использование ее без увеличения времени расчета более 3000 гармоник в целях преодоления эффекта Гиббса $[23,29]$. При расчете происходит последовательное преобразование схемы замещения от конца к началу с применением классических методов расчета электрических цепей. После выполнения расчетов токов и напряжений в различных элементах схемы замещения и преобразования их 
значения из комплексной фрормы в тригонометрическую можно выполнить построение графиков изменения напряжений и токов во времени:

$$
\begin{aligned}
& U_{j}(t)=\sum_{k=1}^{k_{\max }} U_{k j} \sin \left(2 \pi k f_{1} t+\varphi_{k j}\right) ; \\
& I_{j}(t)=\sum_{k=1}^{k_{\max }} I_{k j} \sin \left(2 \pi k f_{1} t+\phi_{k j}\right),
\end{aligned}
$$

где $j$ - индекс соответствующего напряжения или тока в схеме рис. $3 ; k_{\max }-$ число гармоник разложения в ряд Фурье, принятое в расчетах; $\varphi_{k j}$ и $\phi_{k j}-$ фраза напряжения и тока $k$-й гармоники в рассматриваемой точке.

Результаты расчета установившегося режима ЭТКПЧ с использованием разработанной методики представлены на рис. 9, там же приведены опытные осциллограммы, полученные в ходе многочисленных экспериментов с частотой выходного напряжения преобразователя 13-18 кГц.

Анализ осциллограмм позволяет сравнить результаты расчета (рис. 9,а,в) и эксперимента (рис. 9,б,г) в режиме холостого хода (нагрузка на конце линии отсутствует) и под нагрузкой ЭТКПЧ в ходе электротепловой обработки мощностью 2 кВт. Нельзя не отметить, что имеет место хорошее, но неполное соответствие результатов расчета и эксперимента. Оно проявляется в наличии высокочастотных колебаний вблизи нулевого значения напряжения в момент изменения полярности переменного напряжения на расчетной осциллограмме, которые отсутствуют в экспериментальной осциллограмме (рис. 9,б,г) [10, 11].

Такое расхождение может быть обусловлено влиянием нелинейного характера реальной динамической петли гистерезиса материала (фреррита) магнитопроводов силовых трансформаторов [14] (рис. 6), котоpoe в расчетной методике в настоящее время не учитывается.

Одновременно этот фрактор практически не оказывает влияния на параметры установившегося рабочего режима установки [24]. С другой стороны, в некоторых особых случаях, например в режиме холостого хода, игнорирование этого фрактора может привести к погрешностям в вычислениях. Поэтому требуется дальнейшее развитие и уточнение методики расчета установившихся режимов ЭТКПЧ [18].

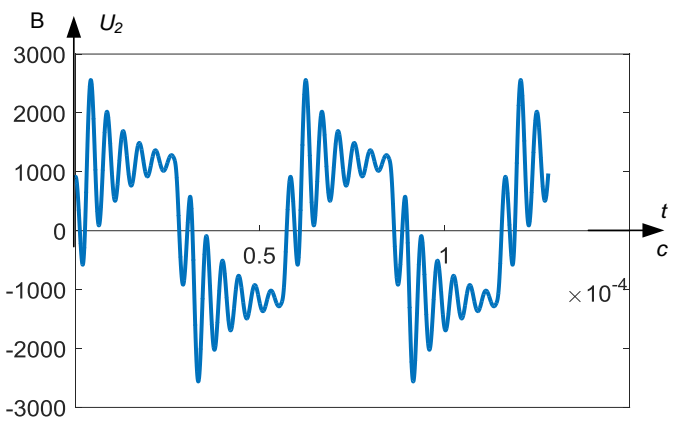

a)

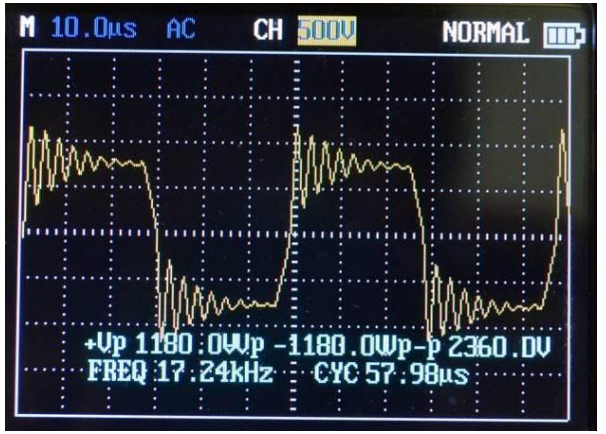

б)

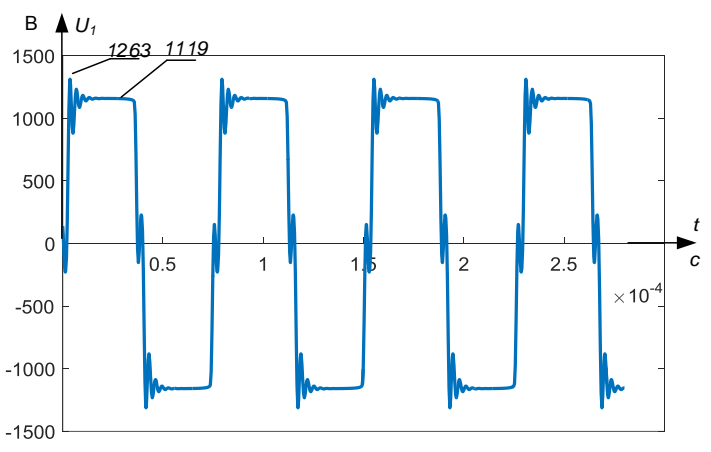

B)

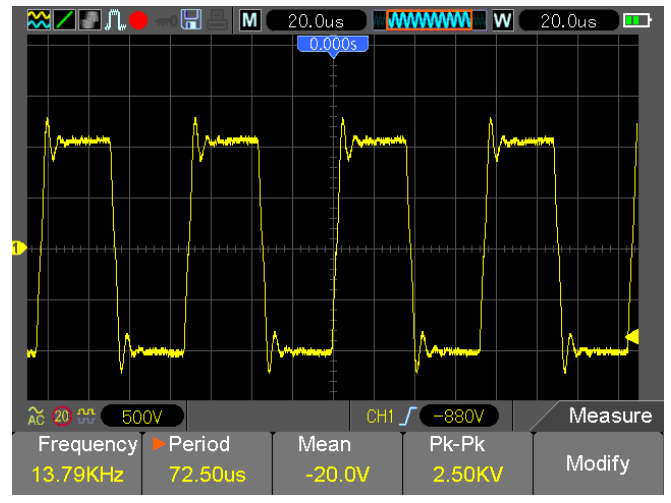

г)

Рис. 9. Сравнение расчетных (а, в) и экспериментальных (б, г) осциллограмм напряжения в конце высоковольтной кабельной линии ЭТКПЧ (рис. 3) в режиме холостого хода $(\mathrm{a}$, б) и под нагрузкой (в, г)

Следует отметить, что получение точной математической модели фрактической динамической петли гистерезиса при- 
менительно к конкретному ферромагнитному материалу и условий его работы представляет собой сложную задачу, которая потребует пересмотра и развития качественных представлений и количественного описания явлений магнетизма ферромагнитных материалов.

Получены результаты автоматизации методики (рис. 9) с применением MATLAB. В отсутствии последнего можно использовать Python. Для создания сложных моделей ЭТКПЧ целесообразно использовать методы имитационного моделирования с применением Simulink, решив при этом несколько дополнительных задач:

- создание программируемого источника ЭДС «трапеция с паузой», так как стандартная библиотека элементов Simulink не содержит блока источника с такой формой напряжения;

- учет влияния поверхностного эффекта в жилах кабельной линии и обмотках трансформатора повышенной частоты.

Выводы. Представленная комплексная методика расчета установившихся режимов несинусоидальных систем переменного тока с различной частотой используемого напряжения является удобным инструментом расчета установившихся режимов систем переменного тока, содержащих инверторы на базе IGBT.

Представленные дополнительные инструменты описания и расчета характеристик компонентов систем переменного тока могут использоваться как вместе, так и по отдельности: инвертор, двухпроводные кабельные линии, силовые трансформаторы повышенной частоты.

Простая и эфффективная реализация методики в настоящее время доступна в среде MATLAB и средствами языков программирования (например, Python с пакетом Math), в то время как создание модели ЭТКПЧ в Simulink требует значительных доработок стандартных элементов библиотеки.

Целесообразно дальнейшее развитие и совершенствование методики расчета установившихся режимов ЭТКПЧ в области учета нелинейных характеристик сердечников силовых трансформаторов повышенной частоты.

\section{Список литературы}

1. Гольдштейн М.Е., Гаврилов А.И. Схемы выдачи мощности и присоединения к энергосистеме «малых» синхронных генерато- ров со скоростью вращения, варьируемой и не обеспечивающей синхронную частоту // Электрооборудование: эксплуатация и ремонт. 2018. - № 4. - С. 52-57.

2. Гольдштейн М.Е., Филяев К.Ю. Взаимосвязь параметров режима энергоблока с синхронным генератором повышенной частоты и полупроводниковым преобразователем частоты // Вестник Южно-Уральского государственного университета. Сер. Энергетика. 2005. - № 9(49). - С. 29-31.

3. Данилевич Я.Б., Сигаев В.Е. Электроэнергетические установки с синхронными генераторами нестандартной частоты // ЭлектричеСтво. - 2000. - № 5. - С. 26-32.

4. Быстроходный турбогенератор для систем нетрадиционной энергетики / Я.Б. Данилевич, И.Ю. Кручинина, В.А. Сапожников и др. // Известия Российской академии наук. Энергетика. -2002 . - № 5. - С. 98.

5. Сверхбыстроходный генератордвигатель для газовых микротурбин / Я.Б. Данилевич, А.В. Иванова, И.Ю. Кручинина, Ю.Ф. Хозиков // Электротехника. - 2004. - № 5. C. 25 .

6. Разработка конструкции и методики проектирования высокочастотных трансформаторов с сердечником из аморфных сплавов / А.И. Тихонов, А.В. Стулов, И.В. Еремин, А.В. Подобный // Вестник ИГЭУ. - 2018. - № 6. C. $57-65$.

7. Разработка пожаробезопасных высокочастотных трансформаторов для зарядных станций мобильного электротранспорта / К.В. Семенова, А.И. Тихонов, А.В. Плаксин, А.А. Каржевин // Пожарная и аварийная безопасность: сб. материалов XIII Междунар. науч.-практ. конф., посвященной Году культуры безопасности. - Иваново, 2018. - С. 218-223.

8. Федосов С.В., Бобылев В.И., Соколов А.М. Электротепловая обработка бетона токами повышенной частоты на предприятиях сборного бетона. - Иваново, 2016. - 336 с.

9. Титов М.М. Технология предварительного электроразогрева бетонной смеси с использованием современного оборудования // Известия вузов. Строительство. - 2009. - № 2. C. 56-62.

10. Особенности применения частотного анализа при расчете электрических цепей с транзисторными преобразователями напряжения / А.В. Гусенков, В.Д. Лебедев, А.М. Соколов и др. // Электричество. - 2016. - № 1. - С. 4-12.

11. Повышение точности и сокращение времени расчета установившихся режимов электротехнических комплексов повышенной частоты / А. Танкой, Т.Е. Шадриков, А.В. Гусенков и др. // Вестник ИГЭУ. - 2019. - Вып. 3. C. $22-31$.

12. Танкой А., Лакеев Д.В. Определение частотных характеристик мощных IGBT транзисторов // (ПОИСК - 2017): межвуз. (с междунар. 
участием) молодежная науч.-техн. конф. «Молодые ученые - развитию текстильнопромышленного кластера». Т. 2. Ч. 2. - Иваново, 2017. - С. 271-273.

13. Методика определения емкостных и индуктивных параметров силовых высоковольтных трансформаторов повышенной частоты / А.В. Гусенков, Т.Е. Шадриков, А.А. Дьячков, А.А. Симакова // Вестник ИГЭУ. - 2016. Вып. 1. - С. 27-33.

14. Вдовин С.С. Проектирование импульсных трансформаторов. - Л.: Энергоатомиздат. Ленингр. отд-ние, 1991. - 208 с.

15. Тихомиров П.М. Расчет трансформаторов: учеб. пособие для вузов. - Изд. 5-е, перераб. и доп. - М.: Энергия, 1986. - 528 с.

16. Evaluation of Frequency and Temperature Dependence of Power Losses Difference in Parallel IGBTs / J. Yang, Y. Che, L. Ran, H. Jiang // IEEE Access. - 2020. DOI: 10.1109/ACCESS.2020.2995971.

17. Розанов Ю.К. Основы силовой электроники. - М.: Энергоатомиздат, 1992. - 296 с.

18. Zhao M.Z., Shi B.C., Zhu Y.C. Control Technologies for Power Electronic Hybrid Systems in High-voltage High-power Applications: A Review // High Voltage Engineering. - 2017. - Vol. 45, no. 7. - P. 2017-2027.

19. A $750 \mathrm{~V}$ recessed-emitter-trench IGBT with recessed-dummy-trench structure featuring low switching losses / Y. Yao, H. Luo, Q. Xiao, Ch. Zhu // IEEE 30th International Symposium on Power Semiconductor Devices and ICs (ISPSD). Chicago, IL, 2018. - P. 112-115. DOI: 10.1109/ISPSD.2018.8393615.

20. Comparative analysis of static characteristics of insulated gate bipolar transistors and thyristors with static induction / O.I. Bonomorskii, A.S. Kyuregyan, A.V. Gorbatyuk, B.V. Ivanov // Russian Electrical Engineering. - 2015. - T. 86, № 2. - C. 93-97.

21. Моделирование статических и динамических потерь в MOSFET-ключах / В.П. Бабенко, В.К. Битюков, В.В. Кузнецов, Д.С. Симачков // Российский технологический журнал. 2018. - T. 6, № 1(21). - C. 20-39.22.

22. Осипов Ю.М. Частотный и временной анализ стационарных и переходных характеристик линейных электрических цепей: учеб. пособие по курсам электротехники и ТОЭ. Ч. 2. СПб.: СПбГИТМО (ТУ), 2002. - 99 с.

23. О возможности и целесообразности физического моделирования электропередачи с нетрадиционными параметрами используемых токов и напряжений / А.В. Гусенков, В.Д. Лебедев, А.М. Соколов, Т.Е. Шадриков // Энергетик. - 2015. - № 4. - С. 29-32.

24. Проектирование линий электропередачи сверхвысокого напряжения / Г.Н. Александров, А.В. Горелов, В.В. Ершевич и др.; под ред. Г.Н. Александрова. - Л.: Энергоатомиздат, 1993. - 530 c.
25. Александров Г.Н. Передача электрической энергии переменным током. - М.: Знак, 1998. - 271 с.

26. Исследование характеристик двухпроводных высоковольтных кабелей для электротехнических комплексов повышенной частоты / Т.Е. Шадриков, А. Танкой, А.М. Соколов и др. // Электротехника. - 2019. - № 8. - С. 53-59.

27. Спектральный анализ электродвижущей силы электроустановки повышенной частоты / А.В. Гусенков, В.Д. Лебедев, А.М. Соколов и др. // Электричество. - 2018. - № 2. C. 17-26.

28. Стариков А.В., Кузнецов В.В., Рокало Д.Ю. Анализ гармонического состава трапецеидального фазного напряжения, формируемого частотным преобразователем // Вестник Самарского государственного технического университета. Сер. Технические науки. - 2017. № 3(55). - С. 75-79.

29. Зырянов В.М., Митрофанов Н.А., Соколовский Ю.Б. Исследование гармонического состава напряжения преобразователя частоты // Электрооборудование: эксплуатация и ремонт. - 2016. - № 9. - С. 34-40.

\section{References}

1. Gol'dshteyn, M.E., Gavrilov, A.I. Skhemy vydachi moshchnosti i prisoedineniya $k$ energosisteme «malykh» sinkhronnykh generatorov so skorost'yu vrashcheniya, var'iruemoy i ne obespechivayushchey sinkhronnuyu chastotu [Schemes of power delivery and connection to the power system of "small" synchronous generators with a rotational speed that is variable and does not provide a synchronous frequency]. Elektrooborudovanie: ekspluatatsiya i remont, 2018, no. 4, pp. 52-57.

2. Gol'dshteyn, M.E., Filyaev, K.Yu. Vzaimosvyaz' parametrov rezhima energobloka s sinkhronnym generatorom povyshennoy chastoty i poluprovodnikovym preobrazovatelem chastoty [The relationship of the parameters of the power unit mode with a synchronous generator of increased frequency and a semiconductor frequency converter]. Vestnik Yuzhno-Ural'skogo gosudarstvennogo universiteta. Seriya Energetika, 2005, no. 9(49), pp. 29-31.

3. Danilevich, Ya.B., Sigaev, V.E. Elektroenergeticheskie ustanovki s sinkhronnymi generatorami nestandartnoy chastoty [Electric power plants with synchronous generators of nonstandard frequency]. Elektrichestvo, 2000, no. 5, pp. 26-32.

4. Danilevich, Ya.B., Kruchinina, I.Yu., Sapozhnikov, V.A., Khozikov, Yu.F., Steinle, L.Yu. Bystrokhodnyy turbogenerator dlya sistem netraditsionnoy energetiki [High-speed turbine generator for non-traditional energy systems]. Izvestiya Rossiyskoy akademii nauk. Energetika, 2002, no. 5, p. 98. 
5. Danilevich, Ya.B., Ivanova, A.V., Kruchinina, I.Yu., Khozikov, Yu.F. Sverkhbystrokhodnyy generator-dvigatel' dlya gazovykh mikroturbin [Ultrafast generator - an engine for gas microturbines]. Elektrotekhnika, 2004, no. 5, p. 25.

6. Tikhonov, A.I., Stulov, A.V., Eremin, I.V., Podobnyy, A.V. Razrabotka konstruktsii i metodiki proektirovaniya vysokochastotnykh transformatorov s serdechnikom iz amorfnykh splavov [Development of the design and design methodology for high-frequency transformers with amorphous alloy cores]. Vestnik IGEU, 2018, issue 6, pp. 57-65.

7. Semenova, K.V., Tikhonov, A.I., Plaksin, A.V., Karzhevin, A.A. Razrabotka pozharobezopasnykh vysokochastotnykh transformatorov dlya zaryadnykh stantsiy mobil'nogo elektrotransporta [Development of fire-safe highfrequency transformers for charging stations of mobile electric transport]. Sbornik materialov XIII Mezhdunarodnoy nauchno-prakticheskoy konferentsii, posvyashchennoy Godu kul'tury bezopasnosti: "Pozharnaya $i$ avariynaya bezopasnost'» [Collection of materials of the XIII International Scientific and Practical Conference dedicated to the Year of Safety Culture: "Fire and emergency safety"]. Ivanovo, 2018, pp. 218-223.

8. Fedosov, S.V., Bobylev, V.I., Sokolov, A.M. Elektroteplovaya obrabotka betona tokami povyshennoy chastoty na predpriyatiyakh sbornogo betona [Electrothermal treatment of concrete by currents of increased frequency at enterprises of precast concrete]. Ivanovo, 2016. $336 \mathrm{p}$.

9. Titov, M.M. Tekhnologiya predvaritel'nogo elektrorazogreva betonnoy smesi s ispol'zovaniem sovremennogo oborudovaniya [Technology of preliminary electric heating of concrete mix using modern equipment]. Izvestiya vuzov. Stroitel'stvo, 2009, no. 2, pp. 56-62.

10. Gusenkov, A.V., Lebedev V.D., Sokolov, A.M., Shadrikov, T.E., Strakhov, A.S. Osobennosti primeneniya chastotnogo analiza pri raschete elektricheskikh tsepey $s$ tranzistornymi preobrazovatelyami napryazheniya [Application of frequency analysis to calculate electrical circuits with transistor voltage converters]. Elektrichestvo, 2016, no. 1, pp. 4-12.

11. Tankoy, A., Shadrikov, T.E., Gusenkov, A.V., Lebedev, V.D., Sokolov, A.M. Povyshenie tochnosti i sokrashchenie vremeni rascheta ustanovivshikhsya rezhimov elektrotekhnicheskikh kompleksov povyshennoy chastoty [Improving the accuracy and time of calculating steady-state modes of increased frequency electrical systems]. Vestnik IGEU, 2019, issue 3, pp. 22-31.

12. Tankoy, A., Lakeev, D.V. Opredelenie chastotnykh kharakteristik moshchnykh IGBT tranzistorov [Determination of frequency characteristics of powerful IGBT transistors]. (POISK - 2017): mezhvuzovskaya (s mezhdunarodnym uchastiem) molodezhnaya nauchno-tekhnicheskaya konferentsiya "Molodye uchenye - razvitiyu tekstil'nopromyshlennogo klastera». T. 2. Ch. 2 [(SEARCH -
2017): Interuniversity (with international participation) youth scientific and technical conference "Young scientists - the development of the textile and industrial cluster". Vol. 2. Part 2]. Ivanovo, 2017, pp. 271-273.

13. Gusenkov, A.V., Shadrikov, T.E., D'yachkov, A.A., Simakova, A.A. Metodika opredeleniya emkostnykh i induktivnykh parametrov silovykh vysokovol'tnykh transformatorov povyshennoy chastoty [Method for determining the capacitive and inductive parameters of highfrequency power transformers]. Vestnik IGEU, 2016, issue 1, pp. 27-33.

14. Vdovin, S.S. Proektirovanie impul'snykh transformatorov [Design of pulse transformers]. Leningrad: Energoatomizdat. Leningradskoe otdelenie, 1991. $208 \mathrm{p}$.

15. Tikhomirov, P.M. Raschet transformatorov [Calculation of transformers]. Moscow: Energiya, $1986.528 \mathrm{p}$.

16. Yang, J., Che, Y., Ran, L., Jiang, $H$. Evaluation of Frequency and Temperature Dependence of Power Losses Difference in Parallel IGBTs. IEEE Access. 2020. DOI: 10.1109/ACCESS.2020.2995971.

17. Rozanov, Yu.K. Osnovy silovoy elektroniki [Fundamentals of Power Electronics]. Moscow: Energoatomizdat, 1992. $296 \mathrm{p}$.

18. Zhao, M.Z., Shi, B.C., Zhu, Y.C. Control Technologies for Power Electronic Hybrid Systems in High-voltage High-power Applications: A Review. High Voltage Engineering, 2017, vol. 45, no. 7, pp. 2017-2027.

19. Yao Y., Luo H., Xiao Q., Zhu Ch. A 750 V recessed-emitter-trench IGBT with recesseddummy-trench structure featuring low switching losses. 2018 IEEE 30th International Symposium on Power Semiconductor Devices and ICs (ISPSD), Chicago, IL, 2018, pp. 112-115. DOI: 10.1109/ISPSD.2018.8393615.

20. Bonomorskii, O.I., Kyuregyan, A.S., Gorbatyuk, A.V., Ivanov, B.V. Comparative analysis of static characteristics of insulated gate bipolar transistors and thyristors with static induction. Russian Electrical Engineering, 2015, vol. 86, no. 2, pp. 93-97.

21. Babenko, V.P., Bityukov, V.K., Kuznetsov, V.V., Simachkov, D.S. Modelirovanie staticheskikh i dinamicheskikh poter' v MOSFETklyuchakh [Modeling of static and dynamic losses in MOSFET switches]. Rossiyskiy tekhnologicheskiy zhurnal, 2018, vol. 6, no. 1(21), pp. 20-39.

22. Osipov, Yu.M. Chastotnyy i vremennoy analiz statsionarnykh i perekhodnykh kharakteristik lineynykh elektricheskikh tsepey [Frequency and time analysis of stationary and transient characteristics of linear electrical circuits]. Saint-Petersburg: SPbGITMO (TU), 2002. 99 p.

23. Gusenkov, A.V., Lebedev, V.D., Sokolov, A.M., Shadrikov, T.E. O vozmozhnosti i tselesoobraznosti fizicheskogo modelirovaniya elektroperedachi s netraditsionnymi parametrami 
ispol'zuemykh tokov i napryazheniy [On Possibility and feasibility of physical modeling of power transmission with unconventional parameters of the used currents and voltages]. Energetik, 2015, no. 4, pp. 29-32.

24. Aleksandrov, G.N., Gorelov, A.V., Ershevich, V.V. Proektirovanie liniy elektroperedachi sverkhvysokogo napryazheniya [Design of ultra-high voltage power lines]. Leningrad: Energoatomizdat, 1993. 530 p.

25. Aleksandrov, G.N. Peredacha elektricheskoy energii peremennym tokom [Transmission of electrical energy by alternating current]. Moscow: Znak, 1998. 271 p.

26. Shadrikov, T.E., Tankoy, A., Sokolov, A.M., Gusenkov, A.V., D'yachkov, A.A., Lebedev, V.D. Issledovanie kharakteristik dvukhprovodnykh vysokovol'tnykh kabeley dlya elektrotekhnicheskikh kompleksov povyshennoy chastoty [Investigation of the characteristics of two-wire high-voltage cables for high-frequency electrical complexes]. Elektrotekhnika, 2019, no. 8, pp. 53-59.

27. Gusenkov, A.V., Lebedev, V.D., Sokolov, A.M., Shadrikov, T.E., Tankoy, A.
Spektral'nyy analiz elektrodvizhushchey sily elektroustanovki povyshennoy chastoty [Spectral analysis of the electromotive force of an electrical installation of increased frequency]. Elektrichestvo, 2018, no. 2, pp. 17-26.

28. Starikov, A.V., Kuznetsov, V.V., Rokalo, D.Yu. Analiz garmonicheskogo sostava trapetseidal'nogo faznogo napryazheniya, formiruemogo chastotnym preobrazovatelem [Analysis of the harmonic composition of the trapezoidal phase voltage generated by the frequency converter]. Vestnik Samarskogo gosudarstvennogo tekhnicheskogo universiteta. Seriya Tekhnicheskie nauki, 2017, no. 3(55), pp. 75-79.

29. Zyryanov, V.M., Mitrofanov, N.A., Sokolovskiy, Yu.B. Issledovanie garmonicheskogo sostava napryazheniya preobrazovatelya chastoty [Investigation of the harmonic composition of the voltage of the frequency converter]. Elektrooborudovanie: ekspluatatsiya $i$ remont, 2016, no. 9, pp. 34-40. 